\title{
Perfecting the use of hybrid models in scaling analysis
}

\author{
F. Reventos*, V. Martinez-Quiroga, J. Freixa \\ Department of Physics and Nuclear Engineering, Universitat Politécnica de Catalunya (UPC), Spain
}

\section{A R T I C L E I N F O}

\section{Keywords:}

Integral test facility

PWR

Scaling

Hybrid

\begin{abstract}
A B S T R A C T
Different methodologies devoted to qualify Nuclear Power Plant (NPP) system-code nodalizations rely on Kvscaled calculations as an essential tool for their purpose. In the framework of Power-To-Volume strategy, a Kvscaled calculation is a simulation in which, defined Integral Test Facility (ITF) test conditions are scaled-up to a NPP nodalization to reproduce the same scenario. In the recent years, the "Universitat Politècnica de Catalunya" (UPC) has developed SCUP, a scaling methodology to qualify NPP models. Following the steps established in UPC's SCUP methodology, Kv-scaled calculations become really useful when two different kinds of them, the socalled "pure scaled" and "hybrid" are properly combined.

This paper starts summarizing the most relevant features of UPC's SCUP methodology and its validation process. It also includes important aspects of the usefulness of hybrid calculations in their role of explaining distortions that may appear when a plant simulation is compared with a scaled-up version of test results.

Some ideas on the forthcoming uses of hybrid nodalizations are also presented. Among such uses the most significant is the support to test design. Hybrid calculations are used to justify facility modifications. This particular, despite being more specific for future modular ITFs, is illustrated in the paper for minor changes in existing facilities.
\end{abstract}

\section{Background and introduction in $\mathrm{Kv}$-scaled calculations}

Addressing the scaling issue refers to a rather complex process having the aim of demonstrating the applicability of activities devoted to predict the behaviour of actual nuclear power plant using the knowledge of the scenario adquired in scaled-down test facilities. Such activities involve among others the evaluation of the capability of $\mathrm{BE}$ codes to scale-up processes from reduced scale test facilities to full scale NPPs and the quantification the effects of scale distortions. Kv-scaled calculations are performed in such context.

In the framework of Power-To-Volume strategy, a Kv-scaled calculation is a system-code simulation in which, defined test conditions of an Integral Test Facility (ITF) are scaled-up to a Nuclear Power Plant (NPP) nodalization to reproduce the same scenario. The practical use of such kind of calculation allows a comparison of the behaviour of the plant and the ITF nodalizations under the same conditions. Different methodologies (D'Auria et al., 1995; Reventós et al., 2007) devoted to validate plant models establish the usefulness of $\mathrm{Kv}$-scaled calculations to check the validity of the NPP nodalization. The concept was introduced by DAuria et al. (1995), used in different contexts (Petelin et al., 2007; Pla et al., 2007; Freixa et al., 2009) and consolidated by Martínez-Quiroga (2014).
The comparison between the NPP Kv-scaled results and those of the experiment post-test calculation shows unavoidable differences or distortions. Explaining such distortions is the key process in methods devoted to qualify plant nodalizations. A variety of techniques are available to fulfil this goal, as shown in OECD State-of-the-Art report on Scaling (Bestion et al., 2017).

The goal of this paper is to show the effectiveness of Kv-scaled calculations and more in detail to outline the forthcoming use of hybrid nodalizations. To do so, the article starts taking account of a brief description of essential aspects of UPC's scaling methodology which include its associated software, its validation process and a few words on its current combination with uncertainty evaluation. Then it introduces some examples of hybrid calculations devoted to qualify NPP nodalization. And finally it illustrates the perspectives of future uses of the developed techniques. Such future uses include the feedback to experimentation. Despite the fact that the hybrid calculations presented here are related to existing ITFs and NPPs, feedback to experimentation intents to show the essentials of a future practice to be mainly implemented in modular ITFs. Modularity, geometry flexibility as well as the possibility of interchanging components in test facilities is still under discussion inside the scientific community but some leading initiatives begin to be a fact as shown by Hyvärinen et al. (2017).

\footnotetext{
* Corresponding author.

E-mail addresses: francesc.reventos@upc.edu (F. Reventos), victor.martinez@upc.edu (V. Martinez-Quiroga), jordi.freixa-terradas@upc.edu (J. Freixa).
} 


\subsection{Methodology}

Based on its own calculating experience and with the aim of consolidating analytical capabilities, the Advanced Nuclear Technologies (ANT) team from UPC prepared its own scaling methodology which is fully described by Martinez-Quiroga et al. (2014), Martinez-Quiroga and Reventos (2014), Martínez-Quiroga (2014). The methodology has been developed to contribute to the qualification of NPP nodalizations by means of scale approaches. The techniques that are presented include the already mentioned Kv-scaled calculation approach as well as the use of "hybrid nodalizations" and "scaled-up nodalizations". These methods have revealed themselves to be very helpful in producing the required qualification and in promoting further improvements in nodalization techniques. In the paper presented by Martinez-Quiroga and Reventos (2014) both the concepts and the general guidelines of the method are thoroughly explained. The main procedure of the methodology can be summarized in the following steps:

1. Select a specific scenario for qualyfying the NPP Nodalization. In this step relevant phenomena must be identified and a set of ITF experiments must be selected. A validation matrix must be generated by relating the experiments with the particular phenomena to be qualified in the selected scenario.

2. Validate the ITF nodalizations and the ITF post-test calculations selected in the NPP validation matrix.

3. Perform a Kv scaled calculation with the NPP nodalization.

4. Analyze and establish the scaling and design effects in the simulation results by using generated scaled-up and hybrid nodalizations.

5. Perform an expert judgment by comparing the results of step (3) combined with conclusions of step (4).

Once the four steps have been completed, if results are not consistent the analyst must improve the NPP nodalization with those modelling remarks obtained from the ITF post-test analysis. Otherwise, if Kv scdaled calculation is consistent with ITF simulations and with the scaling and design effect analysis, the NPP nodalization can be considered as qualified.

The paper gives details on each of these steps along with some results of analytical examples. Since scaled-up and hybrid nodalizations are very significant in the current context, their differences and similarities are emphasized below.

Scaled-up nodalizations are helpful to clarify two important sources of distortion. These are the treatment of environment and passive heat structures and also preserving Froude number in horizontal components. The paper shows different comparisons among results obtained using different nodalizations. An example is shown:

- Sc-up nodalization A: a regular scaled-up nodalization following the Kv factors of the Power-to-Volume scaling method

- Sc-up nodalization B: a regular scaled-up nodalization also preserving environment heat losses

- Sc-up nodalization C: a regular scaled-up nodalization also preserving environment heat losses and Froude Number

Fig. 1 is one of the figures of the paper by Martinez-Quiroga et al. (2014) that contributes to show that scaling-up only produced agreement when heat losses and Froude Number are preserved. In this figure an idealized scaled up nodalization where the heat losses and the Froude number have been preserved is compared to its original nodalization results. Scaled-up nodalizations in which scaling distortions have been identified and minimized are named in the methodology as "ideal scaled-up nodalizations".

In a similar way, the paper deals with Design Effect Analyses. In this step, the analyst must be able to justify the discrepancies that appear in a Kv scaled calculation by means of the differences in design between the ITF and the NPP. In that sense, "hybrid nodalizations" are compared

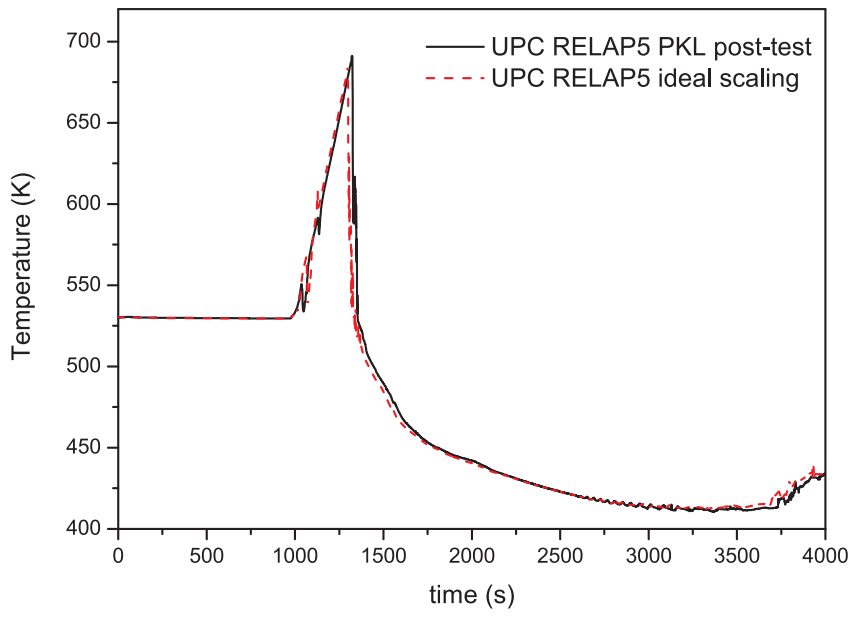

Fig. 1. Core exit temperature obtained with the PKL post-test calculation and the PKL up-scaled model to the LSTF scale.

with the Kv scaled calculation and with the ideal scaled-up nodalization obtained from the scaling effect analyses. Some components of the NPP nodalization are copied and added to the scaled-up nodalization to generate the hybrid decks. Such modifications allow to differentiate the impact of each tested component on the simulation.

Fig. 2 is one of the results of scaling PKL to LSTF. This is equivalent to derive what it is expected to occur in LSTF facility starting from what it actually occurred in PKL, and using only scaling techniques as well as the knowledge of both facilities. In this case PKL hybrid E nodalization is a PKL nodalization scaled up at LSTF scale and including hydrodynamic components, vessel walls heat structures and material properties of LSTF. More details about the complete process can be found in the original paper.

\subsection{PVST tool}

The process of up-scaling complex nodalizations is not trivial and required the generation of a new tool. A specific paper by MartínezQuiroga et al. (2018) introduces the "Power-to-Volume-Scaling Tool" (PVST) to assess scaling distortions associated to code simulations. PVST is a software developed at UPC for generating scaled input decks following the Power to Volume Scaling criterion. Its main purpose is to provide an easy way for obtaining and comparing counterpart

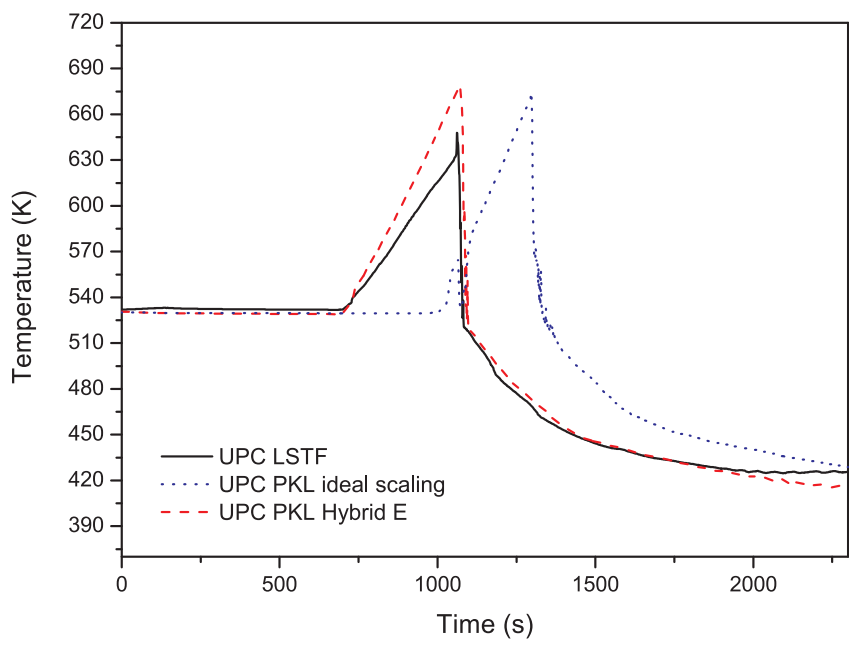

Fig. 2. Core exit temperature. The three lines represent: the LSTF post-test calculation, the PKL idealized up-scaled and the PKL idealized up-scaled model with added hybrid components. 
nodalizations at different scales. It makes it possible to analyze the distortions that may appear between the simulations as a result of the scaling criterion. The tool reads an existing input deck of an ITF and produces a new input deck of a fictitious facility scale-up by a given factor.

Since ITFs are designed with the aim of analyzing the response of a commercial NPP under accidental conditions, the tool has been prepared bearing in mind their design criteria. Thus, for every kind of component the tool finds the parameters that must be scaled, those that must be kept in their original value, and acts consequently. Since the tool depends on the input deck structure, it has been prepared for RELAP5mod3 only.

In the Power-to-Volume strategy, as a general rule, power and volume are scaled while heights are maintained (Navahandi et al., 1979). Consequently, and depending on input structure, cross sections are scaled. Regarding initial and boundary conditions, pressure, temperature and time are kept while mass flow is scaled.

For multi-channel regions, like the core or SG riser, in which the relevant heat exchange processes of the facility take place, it is essential to scale identical flow regimes and heat transfers behaviour independently of the transient conditions. Thus, identical geometries and flow paths are preserved. This consists of keeping the geometries of the NPP channels (heights, hydraulic diameters and pitch-to-rod diameter ratio) but reducing their number by the Kv factor for the ITF and thus preserving the volume scaling criterion.

Two special features are also included in order to facilitate the analysis of distortions. One is related to surface-to-volume ratio and the other to Froude number in horizontal legs. Scaling input decks keeping the surface-to-volume ratio allows preserving the impact of the environment heat losses. Scaling input decks keeping Froude Number value in selected components, allows preserving flow regime transitions in horizontal legs (Zuber, 1980). Both capabilities are helpful when the analyst explores the reason of distortions.

Results demonstrated the high reliability of the software for identifying those particular phenomena (environment heat losses, broken legs flow regime transitions, subcooled liquid vapour mixing, etc) that modify the experiment simulations because of the Power-To-Volume (PtoV) scaling criterion. The software is currently used and maintained by UPC's team.

\subsection{Validating the methodology}

As a contribution to its validation process, SCUP methodology has been applied (Martinez-Quiroga et al., 2014) with the purpose of analyzing discrepancies that appear between the simulations of two counterpart tests. It allows the analysis of scaling-down criterion used for the design of an ITF and also the investigation of the differences of configuration between an ITF and a particular NPP. For analyzing both, it applies the two concepts already introduced "scaled-up nodalizations" and "hybrid nodalizations". The result of this activity is the explanation of appeared distortions and its final goal is to qualify nodalizations for their use in the analysis of equivalent scenarios at an NPP scale. In this sense, the experimental data obtained in the OECD/NEA PKL-2 and ROSA-2 projects as counterpart test are of a great value for the testing of the present methodology. The results of the posttest calculations of LSTF-PKL counterpart tests have allowed the analyst to define which phenomena could be well reproduced by their nodalizations and which not, in this way establishing the basis for a future extrapolation to an NPP scaled calculation. The application of the UPC scaling up methodology has demonstrated that selected phenomena can be scaled-up and explained between counterpart simulations by carefully considering the differences in scale and design.

\subsection{Combining scaling with uncertainty evaluation}

A series of analytical activities combining scaling with uncertainty evaluation has been helpful for the consolidation of SCUP methodology. One of them (Casamor et al., 2019) focuses on the evaluation of the scaling of uncertainties for BEPU methodologies. It is based on Test 3 of the OECD/NEA ROSA-2 project from the LSTF facility. The facility was designed following the power-to-volume scaling approach with a scaling factor of $1 / 48$. The experiment represents a small break loss-ofcoolant-accident at the hot leg. A post-test calculation was performed in the past showing the validity of RELAP5 to reproduce the involved phenomenology. The RELAP5 nodalization has been up-scaled to the Asco NPP reactor size (39/1), and an intermediate scale (25/1) using the PVST. The comparison of the three calculations at different scales provided equivalent results when known scaling distortions like heat losses were omitted. The three calculations have been complemented by an uncertainty analysis. The comparison of the propagation of the uncertainties at different scales provides an insight on the scalability of the uncertainties and on the validation process of BEPU methodologies. Among the different conclusions of the BEPU analysis comparison, it is particularly relevant that the upper limit of the $1 / 1$ scale covers the experimental value with a margin.

\section{Example of application of hybrid nodalizations}

\subsection{Hybrid calculation on EPR-LSTF}

A work prepared at Paul Scherrer Institute (PSI) related to safety evaluations (Freixa and Manera, 2011) of the Olkiluoto-3 EPR nuclear power plant follows some of the relevant steps of the methodology at that time under development. The study includes the development of a model of the plant using the US-NRC TRACE code and a Kv-scaled calculation of an SBLOCA experiment from the ROSA facility. In that analysis, the comparison was purely done with Kv-scaled calaculations and a series of hybrid models but the model of the ROSA facility was not upscaled.

The selected test to qualify the EPR model was Test 6-1 of the NEA/ OECD ROSA 1 programme, an SBLOCA with the break located in the upper head of the reactor pressure vessel. This experiment was selected due to the interest on the scenario and to the fact that the results of a post-test calculation performed previously using the same code showed a close agreement with the experiment (Freixa and Manera, 2010). This step guarantees that the code is capable of reproducing the phenomenology involved in the scenario.

The paper shows the results of different Kv-scaling calculations devoted to point out and analyse appearing distortions. Some of them use "hybrid nodalizations", according to the wording of the methodology at that time under development. The work manages to properly identify and explain most of the distortions. However, at some point into the transient a significant difference between the $\mathrm{Kv}$ calculation and the post-test calculation was detected. In particular, at the moment when the primary pressure fell below the secondary pressure, the EPR model depressurized at a much faster rate. This is a very important event in SBLOCA scenarios and it marks the end of reflux and condensation conditions and the loss of the secondary heat sink. At this time, flashing at the core takes place leading to core uncovery. The depressurization rate of the primary system is a very important parameter at this stage because it will determine how fast the system pressure will reach the accumulator's set-point. In the paper, the authors tried to determine the main contributor to this difference by making use of hybrid nodalizations, although this concept was still not well developed and therefore not used in the publication. The following two hybrid calculations were performed:

- Using different $\mathrm{Kv}$ factors for the power (Kv-factor of 60 ) and the volume of the system $(\mathrm{Kv}=54)$. In the base case a general $\mathrm{Kv}$ factor of 60 was used, however the scaling relation between the LSTF and the EPR reactor is complex, the volume of the RPV of the EPR is 54 times larger than LSTF while the power is 60 times larger. In this 


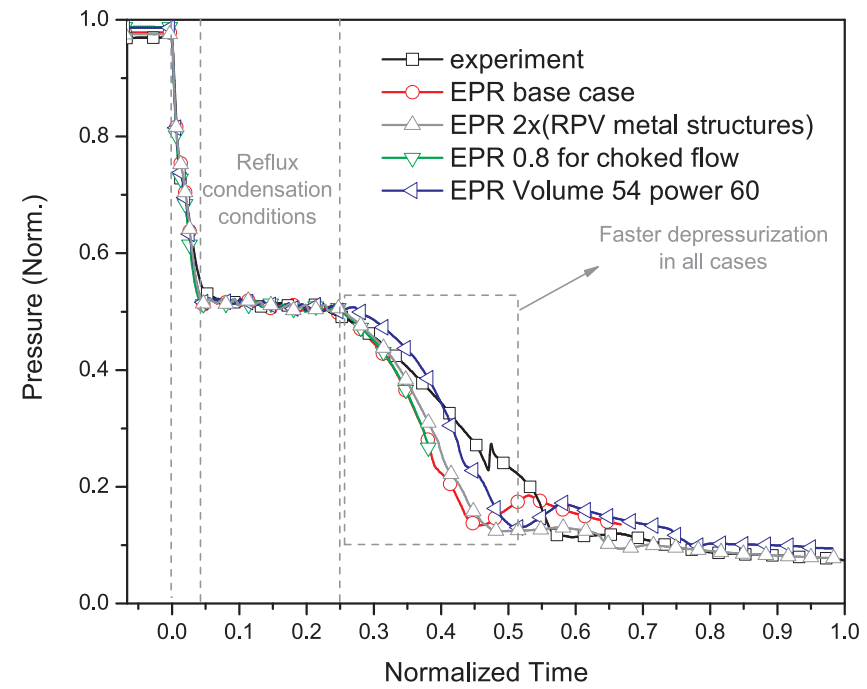

Fig. 3. Primary pressure obtained with the EPR model and different hybrid calculations with the LSTF model. None of the approaches could be explanatory of the higher depressurization rate taking place after the cease of the reflux and condensation conditions. This figure is courtesy of PSI (Freixa and Manera, 2010).

sensitivity this distortion is checked by adjusting the power with a $\mathrm{Kv}$ of 60 and the volume related values (break size and ECC injection) to a scaling $\mathrm{Kv}$ of 54 .

- Doubling the internal passive structures. The internal passive heat structures release heat to the system during a depressurization process. The volume of the internals of the EPR reactor is 26 times larger, thus considering the volumetrical scaling factor. The LSTF facility has more than double of internal structures. By doubling the volume and heat transfer area of the internal heat structures we can evaluate the effect of this distortion.

In addition to the hybrid calculations, the choked flow coefficients at the break location were also tested in order to rule out this important contributor to the faster depressurization. The results of these sensitivities are shown in Fig. 3.

None of these calculations explained the significant difference between the models. A final hypothesis was made about the volume of water below and above the reactor coolant line, the center line of the hot and cold legs at the connection with the RPV. It was demonstrated that the volume of water and steam in the system could alter the rate of depressurization. However, at that time the hypothesis was not demonstrated through a hybrid model but through a stand-alone vessel component were different depressurization rates had been evaluated as a function of the liquid inventory in that vessel.

It was a conclusion of the publication that the faster depressurization was a consequence of having different mass inventories at the starting of the depressurization. Fig. 4 shows the primary pressure for the ROSA post-test calculation compared to the $\mathrm{Kv}$-scaled calculation of the EPR model. It also shows the mass inventory and with a detail of the moment at which, depressurization starts. The detailed graph in the middle shows the stand-alone calculations using a vessel component and how the mass inventory present in the system affects the rate of depressurization.

\subsection{Additional hybrid calculation related to EPR analysis}

If the complete SCUP methodology was to be applied to this particular qualification of the EPR model, an idealized scaled-up model of LSTF should have been created for the TRACE code in order to avoid any distortion related to the scale. In addition, further hybrid nodalizations would have been required until the distortions would be

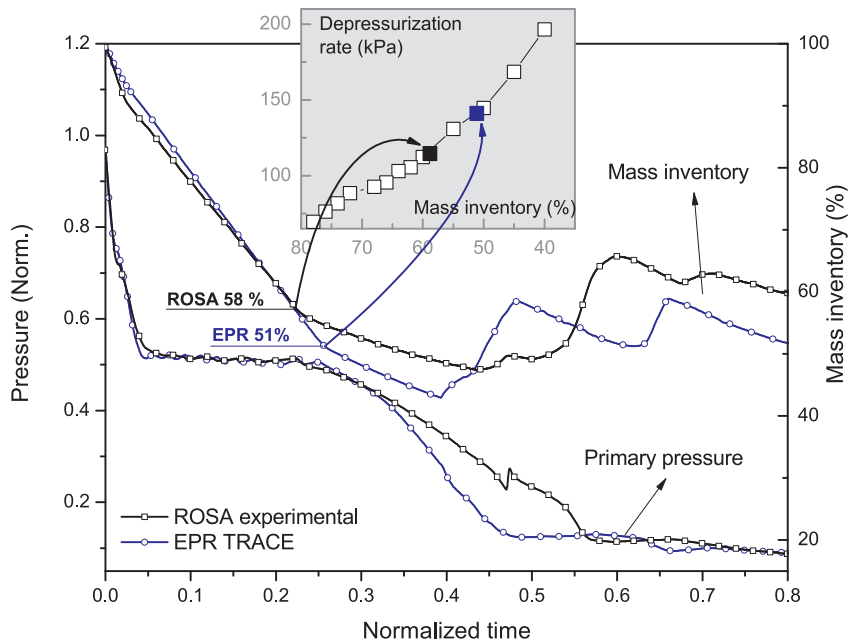

Fig. 4. Primary pressure and mass inventory for the EPRTM model (heat structures volume doubled) and the ROSA experiment. The difference in the depressurization rate occurs right after the interruption of the reflux condenser mode, which is represented by an inflection on the mass inventory curve. This inflection occurs at different mass inventories for the ROSA and EPR models leading to a higher depressurization rate in the EPR simulation. The upper right box shows depressurization rates as function of the mass inventory at the same pressure for a stand alone RPV model. This figure is courtesy of PSI (Freixa and Manera, 2011).

completely explained. In the present analysis, different hybrid models have been created to further evaluate this distortion. 4 different hybrid models have been created with the ROSA model:

1. In order to evaluate the effect of the free volume for the steam, the PZR volume was increased although the initial mass of liquid water was maintained.

2. A tank was connected to the top of the Reactor Pressure Vessel (RPV). The tank was filled with saturated steam at the same pressure conditions as in the primary system at the moment of the start of the depressurization. The volume of the tank was calculated to compensate the differences in Distribution of volumes between ROSA and EPR.

3. The third hybrid calculation consisted of a reduced volume of the LP and DC parts together with an increased volume of the UH. The changes were made to obtain the same proportions as in the EPR design. It is important to notice that this difference were significant ( $7 \%$ of the whole RPV reduction in the LP).

4. The primary side of the steam generator volume was increased by $15 \%$.

The purpose of running the same calculation using these hybrid models is to help exploring the completeness of the explanation given to the appeared distortion.

The results of these two hybrid calculations in comparison to the original post test calculation are shown in Fig. 5. Only the hybrid model with a redistribution of volumes in the RPV showed a faster depressurization in the primary system. This calculation corroborates qualitatively the results of the previous study presented by Freixa and Manera (2011) but shows also that the suggested root cause is not giving the full explanation of the distortion because the depressurization in the EPR model was even faster. This result indicates that either further hybrid analysis should be performed or that the EPR nodalization is not qualified. In this second possible outcome, the model might contain errors in the input data or in the modelling approach. 


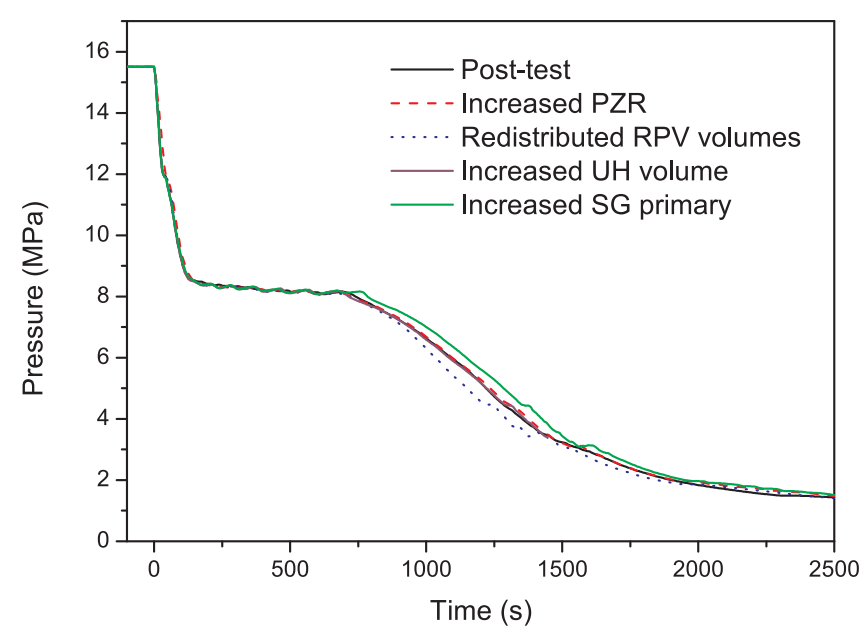

Fig. 5. Primary pressure for the LSTF Test 6-1 post test calculations and further hybrid models that were generated in order to explain the distortions between the LSTF model and the EPR model.

\subsection{Hybrid calculations on AscóNPP-LSTF}

Another paper (Freixa et al., 2016) shows a complete application of the principles established in SCUP methodology and includes different hybrid calculations.

The scaling-up methodology SCUP presented in a previous section has been used in the summarized study to qualify the full scale nodalization of Ascó NPP devoted to reproduce safety phenomena related to the effectiveness of Core Exit Temperature (CET) as an Accident Management (AM) indicator. In order to apply the methodology, the OECD/NEA ROSA-2 Test 3, a SBLOCA in the hot leg, has been selected as a starting point. This experiment was conducted at the Large Scale Test Facility (LSTF) a facility operated by the Japanese Atomic Energy Agency (JAEA) and was focused on the assessment of the effectiveness of AM actions triggered by CET measurements.

The work starts showing the post-test calculation which is a very important step in the SCUP methodology, if the post-test calculation is not sufficiently accurate no further analysis can be performed. Fig. 6 focuses in one single result, the primary pressure, to illustrate the whole process. Subplot a) of Fig. 6 shows the comparison between the experiment and the RELAP5 post-test simulation. The second step is the application of the PtoV tool to upscale the nodalization of the facility to the scale of the Ascó NPP. This is called in the methodology an idealized pure scaling, the adjective idealized is added because in this step the heat losses and other known scale distortions are preserved. The main goal of this step is to evaluate the distortions induced purely by the scale. In that particular case, it was seen that the pure scaling implicated minimal distortions (see subplot b) in Fig. 6).

The paper explains also the improvements performed in RELAP5 Ascó NPP input deck related to lessons learned in nodalizing tasks carried out in LSTF post-test. Such improvements had to do with: multichannel approach, detailed definition of the upper core plate heat structure, simulation of thermocouple heat structures and the definition of hydraulic diameter in core junctions. The aforementioned learned lessons were described in detail by Freixa et al. (2015). Under these conditions a first approach of scaled calculation is performed and the results are compared with those of LSTF scaled-up (see subplot c) in Fig. 6. Some distortions are identified.

The Ascó Kv scaled shows a faster decrease in primary pressure and as a consequence core heat up comes also before and so does the injection from the accumulators.

According to SCUP methodology, the explanation of the identified distortions could be carried out using hybrid calculations, so the continuation of the study consists in series of such calculations. As established in the methodology hybrid nodalizations are prepared adapting the up-scaled ITF nodalization to the configuration of the real plant in the affected part by each considered phenomenon. Hot leg Froude number, RPV bypasses and SG primary volume are most relevant candidates to explain distortions. The subplot d) in Fig. 6 shows the adequacy of selecting them.

Once the distortions are explained, the paper concludes that the tested nodalization is valid to analyse the scenario. The final step is then to use Ascó input deck with the boundary conditions expected in the power plant with the same break size and location but with the core power and Emergency Core Cooling (ECC) dimensioning of the Ascó NPP.
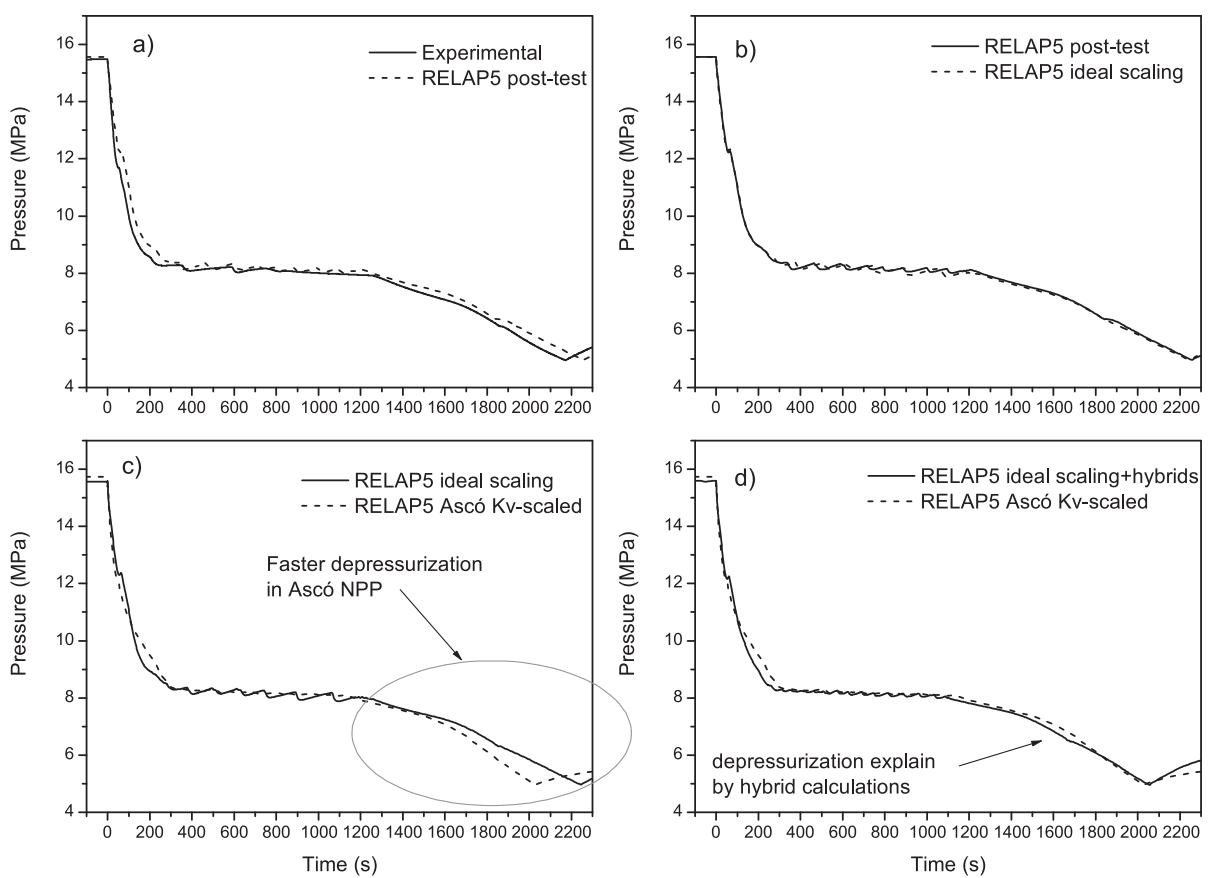

Fig. 6. The four subplots in this figure show the comparison of different hybrid models at the same scale. a) Experimental data compared to the RELAP5 post test. b) RELAP5 LSTF post-test compared with the ideal upscaled model (to the scale of AscóNPP). c) RELAP5 LSTF ideal scaling compared to the RELAP5 Ascó NPP model. d) RELAP5 LSTF ideal scaling + hybrid configuration compared to the RELAP5 Ascó NPP model. 


\section{Forthcoming roles of scaled calculations. The use of hybrid models}

As described in previous sections, scaling analyses include explanations on distortions appeared between results of calculations performed at different scale. Such justifications normally use sensitivity calculations to show the root cause of the different behaviour. Many times, results are considered good enough to explain the distortion and the following step is to rely on modelling techniques to complete the needed prediction at NPP scale.

In many other situations, the root cause of distortions is not easy to isolate and additional analytical work has to be performed to evaluate the magnitude of cause-effect relationship or even to discard any rationale that could apparently explain the distortion in a first approach. Exploring the capabilities of the use of such hybrid models has become an issue following the guidelines established in SCUP methodology.

Such exploring activities are showing some significant advantages that appear as a result of applying the methodology and particularly hybrid models. Besides increasing the usefulness already shown in characterizing and explaining scaling distortions, the results of hybrid calculations are also helpful in two different fields: the support to test design or feedback to experimentation and the contribution to model qualification.

\subsection{Support to test design using hybrid calculation results}

Current situation of integral test facilities (ITFs) in the world has to be taken into account in order to estimate this mentioned usefulness. For this particular, one must distinguish between the existing ones and future builts.

Facing existing ITFs, today a few of them are still operating, and important activities are carried out to share efforts and results obtained in the existing facilities. OECD-NEA is taking care of coordination tasks devoted to support experimental series to be launched in current ITFs like PKL in Germany, LSTF in Japan or ATLAS in Korea. Among such supporting tasks, analytical activities are especially significant. Experiments are nowadays designed after considering different calculations performed by working groups of different countries, discussing their results and reaching an agreement on the scenario and boundary conditions. Considered calculations are related to both ITFs and NPPs and they obviously involve scaling concerns. From now on, and in the context of existing ITFs the concept of reference plant almost belongs to the past. Today experimental results have to be extrapolated to different kinds of plant that could be similar, but not identical, to the original reference plant justifying long ago the first erection of the facility. The analytical support community is taking care of this particular and in many situations is highly motivated by the fact that new facilities are expensive.

Considering the construction of future test facilities, very few proposals exist by now. The one presented by Hyvärinen et al. (2017) is particularly interesting since it shares many of the principles referred in this article. Such facility is, by now, being planed in Finland and it is intended to cope with experiments valid for diferent kinds of reactor, among them: VVER-440, ABWR, EPR and AES-2006 or even SMRs.

In a non-negligible amount of situations, hybrid model results could suggest minor modifications for existing facilities or practical use of modularity for future ones. Implementing physically such modification in the ITF and re-launching again the same test in the reformed facility, could help understanding involved phenomena that could be crucial for the scenario. We are talking about versatile, easy to implement and easy to remove modifications. Obviously, the practicability of the whole thing depends on the feasibility of the modification in terms of cost and operation. Another important question to be considered is related to the expected results of the implementation. Expensive modifications will only be reasonably feasible in case they explain the analysed distortion.

To illustrate how the use of hybrid calculations could feedback experimentation, let us consider the scenarios introduced in previous section related to both EPR and Ascó NPP. Such considerations, based on the knowledge adquired and the availability of data of the involved ITF and NPPs, could help determining the usefulness of such results in testing strategies.

For the EPR case, it seems difficult to suggest any facility modification that could become helpful for consolidating the outcome of hybrid calculations. The two calculations performed in the original paper (Freixa and Manera, 2011) had the goal of discarding direct explanations. Neither using different Kv factors for the power or volume, nor doubling the internal passive structures, seem to be a good recommendation for implementing facility modifications. The other two calculations, those performed specifically for the present study, are in a similar situation. On the one hand connecting a tank or reducing/increasing RPV internal relative volumes seems a rather complex modification. On the other hand the results of hybrid calculations did not fully explain the appeared distortion.

Things are different facing the hybrid calculations related to the second scenario analysed for Ascó NPP (Freixa et al., 2016) also summarized in a previous section. The combined effect of three causes explained the distortions in this case (subplot d) in Fig. 6. Such sources of distortion were:

- Froude number in hot legs

- RPV bypass paths configuration

- Reducing volume of primary loops

The corresponding facility modifications are important but look feasible. That related to Froude number would force to connect a new hot leg with a new value of $\sqrt{L / D}$. That of RPV bypasses would need changes in RPV but there is some experience of similar changes in the past. Finally, that of reducing volume of primary loops is equivalent to plug some SG tubes.

The implementation of such modifications would need further analysis and obviously a close cooperation with the Operating Agent of the facility.

The fact of referring to the combined effect of three sources of distortion needs a comment. In cases in which a single source is identified, everything looks easy to show and demonstrate. These simple cases are not frequent and most of the times one has to deal with two or three (like now) causes at a time. SCUP methodology (MartínezQuiroga, 2014) establishes how calculations have to be combined in order to avoid compensating errors.

If modifications are feasible and a new test is launched, new experimental data help substantially extrapolation to NPP scale.

\subsection{Perfecting model qualification}

The qualification of Nuclear Power Plant nodalizations by means of scale considerations is central goal of SCUP methodology. Among the concepts and techniques introduced $\mathrm{Kv}$-scaled calculations and particularly the conceptions of "hybrid nodalizatios" and "scaled-up nodalizations" have been shown as specially significant.

When a distortion between an idealized pure scaled ITF simulation and the corresponding NPP one is fully explained by one or several hybrid calculations, it is considered that NPP nodalization is qualified for its use in the scenarios including the phenomena identified in the previous steps of the method. Thus, SCUP considers hybrid calculations good enough to explain distortions and relies on modelling techniques to complete the needed prediction at NPP scale. In summary, one empirical reference wrapped by several hybrid calculations contributes significantly to this qualification aspect.

Once hybrid calculations are used to feedback experimentation, in the way it is suggested in the previous section, new empirical evidence is produced on closed scenarios is this will result on perfecting the level of qualification of the final NPP prediction. 


\section{Conclusions}

The paper has shown important aspects of the usefulness of $\mathrm{Kv}$ scaled calculations along with some ideas on the forthcoming uses of hybrid nodalizations. The article started describing the essentials of UPC's SCUP methodology and cited the most relevant results produced so far.

The main part of this study is devoted to hybrid calculations, the way they are currently used to qualify NPP nodalization, and the perspectives of future uses in the framework of the developed methodology.

The article manages to show how the correct choice of hybrid calculations allows advancing in the knowledge of both the behaviours observed empirically and those simulated using models.

Simulations justifying facility minor modifications are also presented. It is a different and innovative use of hybrid calculations that will for sure have future concern in feedback to experimentation. Examples on such use for existing facilities are shown with interesting results.

\section{Acknowledgements}

This work was partly funded by the Spanish Safety Council CSN (Consejo de Seguridad Nuclear). The authors are grateful to the Paul Scherrer Insititute who permitted to perform some additional hybrid calculations with the TRACE LSTF model. This paper contains findings that were produced within the NEA/OECD-PKL-3 and NEA/OECDROSA-2 Projects.

\section{References}

Bestion, D., D'Auria, F., Lien, P., Nakamura, H., 2017. A State-of-the-Art Report on Scaling in System Thermal-Hydraulics Applications to Nuclear Reactor Safety and Design. CSNI.

Casamor, M., Martínez-Quiroga, V., Reventós, F., Freixa, J., 2019. On the scaling of uncertainties in thermal hydraulic system codes. Ann. Nucl. Energy.

D'Auria, F., Debrecin, N., Galassi, G.M., 1995. Outline of the uncertainty methodology based on accuracy extrapostion (UMAE). Nucl. Technol. 109, 21-38.

Freixa, J., Manera, A., 2010. Analysis of an RPV upper head SBLOCA at the ROSA facility using TRACE. Nucl. Eng. Des. 240 (7), 1779-1788.

Freixa, J., Manera, A., 2011. Verification of a TRACE EPR model on the basis of a scaling calculation of an SBLOCA ROSA test. Nucl. Eng. Des. 241 (3), 888-896. URL:http:// www.sciencedirect.com/science/article/pii/S0029549311000148.

Freixa, J., Martínez-Quiroga, V., Reventós, F., 2016. Qualification of a full plant nodalization for the prediction of the core exit temperature through a scaling methodology. Nucl. Eng. Des. 308, 115-132. URL:http://linkinghub.elsevier.com/retrieve/pii/ S0029549316302680.

Freixa, J., Martínez-Quiroga, V., Zerkak, O., Reventós, F., 2015. Modelling guidelines for core exit temperature simulations with system codes. Nucl. Eng. Des. 286, 116-129. URL:http://linkinghub.elsevier.com/retrieve/pii/S0029549315000850.

Freixa, J., Reventós, F., Pretel, C., Batet, L., Sol, I., 2009. SBLOCA with boron dilution in pressurized water reactors. Impact on operation and safety. Nucl. Eng. Des. 239 (4), 749-760.

Hyvärinen, J., Telkkä, J., Kauppinen, O., Purhonen, H., 2017. Motel: modular design and physical scaling principles of the next generation thermal hydraulics test facility at LUT. In: NURETH-17.

Martínez-Quiroga, V., Freixa, J., Reventós, F., 2018. PVST, a tool to assess the Power to Volume scaling distortions associated to code simulations. Nucl. Eng. Des. 332.

Martinez-Quiroga, V., Reventos, F., 2014. The use of system codes in scaling studies: relevant techniques for qualifying NPP nodalizations for particular scenarios. Sci. Technol. Nucl. Install. 1-13. URL:http://www.hindawi.com/journals/stni/2014/ 138745/.

Martinez-Quiroga, V., Reventos, F., Freixa, J., 2014. Applying UPC scaling-up methodology to the LSTF-PKL counterpart test. Sci. Technol. Nucl. Install. 1-18. URL:http://www.hindawi.com/journals/stni/2014/292916/.

Martínez-Quiroga, V., 2014. Scaling-up methodology, a systematical procedure for qualifying NPP nodalizations. Application to the OECD/NEA ROSA-2 and PKL-2 Counterpart test (Ph.D. thesis). Universitat Politécnica de Catalunya.

Navahandi, A., Castellana, S., Moradkhaniav, E., 1979. Scaling laws for modeling nuclear reactor systems. Nucl. Sci. Eng. 72 .

Petelin, S., Mavko, B., Koncar, B., Hassan, Y.A., 2007. Scaling of the small-scale thermalhydraulic transient to the real nuclear power plant. Nucl. Technol. 158 (1), 56-68.

Pla, P., Reventós, F., Pretel, C., Giannotti, W., D’Auria, F., Annunziato, A., 2007. Code validation and scaling of the LOBI BL-30 experiment. In: ICCAP 2007. Nice, France.

Reventós, F., Batet, L., Llopis, C., Pretel, C., Salvat, M., Sol, I., 2007. Advanced qualification process of ANAV NPP integral dynamic models for supporting plant operation and control. Nucl. Eng. Des. 237 (1), 54-63. URL:http://www.sciencedirect.com/ science/article/pii/S0029549306003694.

Zuber, N., 1980. Problems in Modeling of Small Break. LOCA. 